Medico, Enzo

[55]

\section{A genome-wide trap screening procedure for transcriptionally responsive genes}

\author{
Enzo Medico ${ }^{1,2}$, Alessandra Gentile ${ }^{1}$, Philippe Soriano ${ }^{2}$ \\ \& Paolo Comoglio ${ }^{1}$
}

${ }^{1}$ Institute for Cancer Research and Treatment, University of Torino, Torino, Italy ${ }^{2}$ Fred Hutchinson Cancer Research Center, Seattle, Washington, USA

To identify genes whose expression is regulated by exogenous stimuli, we developed a gene trap strategy for high-throughput functional screening. We built a promoterless retroviral vector carrying the sequence of a green fluoresecnt protein-nitroreductase fusion protein downstream from a splice acceptor site. Fluorescence-activated cell sorting analysis of the transduced population allows identification and sorting of cells in which the trap is integrated downstream from an active promoter. Conversely the nitroreductase moiety allows pharmacological selection against constitutive green fluoresecnt protein-nitroreductase expression. Using hepatocyte growth factor stimulation of liver cells as a model, we combined stimulus administration with either positive or negative selection, to recover cells carrying traps in induced or suppressed genes, respectively. The two selection procedures yielded cell populations whose fluorescence consistently increased or diminished after ligand stimulation. Several distinct responsive clones were isolated, and regulated expression of the trapped gene was confirmed at the RNA level. Responsive clones make a library of reporter cells in which the transcriptional control of the trapped genes can easily be further characterized. This selection procedure allows fast and efficient isolation and characterization of genes whose transcription is regulated by exogenous stimuli in a given cell line.

Mendez, Eduardo

\section{Genomic-scale genetic expression profiles of oral cancers}

\author{
Eduardo Mendez ${ }^{1}$ \& Chen $\mathrm{Chu}^{2}$ \\ ${ }^{1}$ Department of Otolaryngology and Head and Neck Surgery, University of \\ Washington, Seattle, Washington, USA \\ ${ }^{2}$ Fred Hutchinson Cancer Research Center, Seattle, Washington, USA
}

At present classification of oral squamous cell carcinoma depends heavily on pathological examination of tissue. Cancerous tissue is classified according to different morphological and histological characteristics that are used not only to diagnose but also to predict outcome. Yet we know that potentially heterogenous tumors are lumped into single groups when they could have even subtly different degrees of aggressiveness. Using oligonucleotide arrays, we have classified oral cavity squamous cell carcinomas by generating genomic-scale genetic expression profiles at different histopathological grades. We compared expression intensities for each gene represented in the oligonucleotide array from tumor tissues at different neoplastic stages with those of normal tissue. We then reported the differences in expression as fold changes in intensity for each gene. Our data, generated using hierarchical clustering analysis, not only confirm previous findings on oral cancer gene expression but also show specific patterns that seem to cluster with each histopathological grade studied. Well-differentiated tumors and moderately differentiated tumors show different patterns of expression. Moreover, using gene cluster analysis, we observed heterogeneity in genetic expression profiles among cancers of a particular histopathological grade. In addition, several genes show overexpression throughout all stages of neoplasia studied, whereas others seem to be downregulated uniformly. We were also able to identify genes that show upregulation as the histopathological grading worsens. The implications of these findings for clinical outcome prediction, the discovery of new tumor markers and the generation of genomic-scale genetic expression databases for oral cancers will be discussed.

Merajver, Sofia

[57]

\section{Microarray analysis of RhoC-transformed mammary epithelial cells suggests molecular mechanisms of inflammatory breast cancer}

Sofia Merajver ${ }^{1}$, Zhi-Fen Wu ${ }^{1}$, Tammy Chang ${ }^{1}$, Hamid Mirshahidi ${ }^{2}$, Paul Meltzer ${ }^{3} \&$ Kenneth vanGolen ${ }^{1}$

${ }^{1}$ University of Michigan, Ann Arbor, Michigan, USA

${ }^{2}$ St. Joseph's Mercy Hospital, Ann Arbor, Michigan, USA

${ }^{3}$ National Human Genome Research Institute, National Institutes of Health, Bethesda, Maryland, USA

Rhoc GTPase is a multifunctional member of the family of ras homology proteins, which are involved in cytoskeletal organization and function. Previous studies of the genetic determinants of inflammatory breast cancer (IBC) revealed that Rhoc is overexpressed in $91 \%$ of IBC tumors but not in non-IBC tissue. We subsequently showed that RhoC is a transforming oncogene for immortalized mammary epithelial cells (HME), generating a highly invasive and angiogenic phenotype akin to IBC. We proposed that $\mathrm{RhoC}$ overexpression induces expression of genes for cytoskeletal activity, motility and angiogenic mediators. In order to analyze the pattern of gene expression stimulated by RhoC overexpression, we compared Rhoc-transfected mammary epithelial cells with cells that had not been transfected, using a microarray of 5,000 complementary DNAs. Rhoc induces the overexpression (7.88- to 1.45 -fold) of 29 genes and decreases (0.8- to 0.3 -fold) the expression of 8 genes. RhoC induces expression of key cytoskeletal structural genes, cell adhesion genes, membrane-associated genes, genes that control intracellular metabolism and genes involved in mediating angiogenesis. The pattern of gene expression suggests a model for signaling mechanisms activated by this important oncogene through activation of the cytoskeleton and motility. Tests of biological activity of several of these key genes reveal specific roles in the malignant phenotype induced by RhoC, further supporting the hypothesis that their expression contributes to invasion and angiogenesis. This work suggests specific therapeutic interventions for IBC. 